\title{
Efficacy of Intra-Articular Injection of Corticosteroids Combined with Local Anesthetics in the Management of Temporomandibular Joint Pain: A Retrospective Study

\author{
Chitra Priya Emperumal' ${ }^{1}$, BDS, MS, Yuka Abe ${ }^{1,2}$, DDS, PhD, Mariela Padilla ${ }^{1,3 *}$, DDS, M.Ed, \\ Reyes Enciso ${ }^{4}$, PhD, Glenn T. Clark ${ }^{1}$, DDS, MS \\ ${ }^{1}$ Orofacial Pain and Oral Medicine Center, Herman Ostrow School of Dentistry, University of Southern \\ California, Los Angeles, California, USA. \\ ${ }^{2}$ Department of Prosthodontics, School of Dentistry, Showa University, Tokyo, Japan.
} \\ ${ }^{3}$ Division of Periodontology, Diagnostic Sciences and Dental Hygiene, Herman Ostrow School of Dentistry, University of Southern California, Los Angeles, California, USA. \\ ${ }^{4}$ Division of Dental Public Health and Pediatric Dentistry, Herman Ostrow School of Dentistry, University of Southern California, Los Angeles, California, USA. \\ *Corresponding Author: Mariela Padilla, DDS, M.Ed, Herman Ostrow School of Dentistry, University of Southern California, 925 W. 34th Street, Los Angeles, California 90089-0641, USA.
}

\section{Abstract}

Background and Objectives: Although intra-articular injection is considered one of the therapeutic options for temporomandibular joint (TMJ) pain, it is not well understood whether in combination with local anesthetics, it is effective for TMJ pain. The objective of this study is to determine the efficacy of intra-articular injections of corticosteroids combined with local anesthetics in patients with TMJ pain and identify predictors for pain improvement.

Methods: Charts of patients with persistent TMJ pain and a diagnosis of TMJ disorders who underwent intraarticular injections of $1.5 \mathrm{ml}$ containing $20 \mathrm{mg}$ triamcinolone and 10 or $20 \mathrm{mg}$ lidocaine were retrospectively analyzed. The TMJ pain intensity on palpation before treatment, immediately after injection, and at follow-up was assessed using a verbal Numerical Rating Scale (NRS).

Results: Thirty-seven injections of 31 patients were identified. The pre-treatment pain NRS score (median=7, interquartile range: 5-8) was significantly reduced immediately after injection (median $=0$, interquartile range: $0-0, p<0.001$ ) and at follow-up (median $=3$, interquartile range: $0-6, p<0.001$ ). Although transient facial palsy (2 cases) and bite change (1 case) were found after injection, no significant complications were reported. Greater pain improvement at follow-up was associated with cases without osteoarthritis ( $p=0.004)$. In addition, pain improvement was modestly correlated with the pre-treatment pain score (Spearman's rho $=0.328, p=0.048$ ) and immediate pain relief after injection (Spearman's rho $=0.375, p=0.022$ ).

Conclusion: Intra-articular injections of corticosteroids in combination with local anesthetics may contribute to favorable prognosis of TMJ pain, with the absence of osteoarthritis serving as one of the predictors of TMJ pain improvement. The results highlight that pre-treatment conditions and diagnoses play a relevant role in the prognosis of TMJ pain after intra-articular injection.

Keywords: temporomandibular joint disorders, orofacial pain, intra-articular injections, corticosteroids, local anesthetics, pain measurement. 
Efficacy of Intra-Articular Injection of Corticosteroids Combined with Local Anesthetics in the Management of Temporomandibular Joint Pain: A Retrospective Study

\section{INTRODUCTION}

Temporomandibular disorders (TMD) are considered a heterogeneous group of musculoskeletal and neuromuscular disorders that embrace pain or dysfunction of the temporomandibular joint (TMJ) complex, and surrounding musculature and osseous components (1). TMD-related pain can interfere with the individual's daily activities, psychosocial functioning, and oral health quality of life $(1,2)$.

The Diagnostic Criteria for Temporomandibular Disorders (DC/TMD) classified TMJ disorders into joint pain, joint disorders, and joint diseases $(1,3)$. The development of TMJ pain could be related to disc displacement, cartilage or joint damage due to arthritis and trauma, parafunction, unstable occlusion, functional overloading, and increased joint friction (4). In line with diagnoses, TMD disorders can be managed with patient education, physical therapy, medications such as pain relievers, anti-inflammatory drugs, or muscle relaxants, oral appliances, corticosteroid injections, and surgical approaches (2).

Regarding intra-articular injections, clinicians can administer different agents, such as sodium hyaluronate, platelet-rich plasma (PRP), plateletderived growth factors (PDGF), corticosteroids alone or in combination with sodium hyaluronate or local anesthetics (5). Corticosteroids are potent antiinflammatory drugs, but intra-articular injections $(6,7)$ have been recommended on a limited basis in case of an acute flare-up of severe joint pain, where conservative therapies (e.g., counseling, avoidance, physiotherapy, oral appliances, anti-inflammatories) have not been successful (2). Sodium hyaluronate is beneficial for pain relief in patients with TMJ osteoarthritis and disc displacement (8). A metaanalysis demonstrated significance of hyaluronic acid injections than corticosteroid alone or in combination with hyaluronic acid after arthrocentesis in reducing pain intensity (5). PRP or PDGF injections have been reported to be effective in the improvement of pain intensity and mandibular range of motion related to TMJ osteoarthritis, disc displacement $(9,10)$, and osteoarthrosis (11).

In medical science, injections of a combination of corticosteroids and local anesthetics can be applied to osteoarthritis or arthritis of various joints for both diagnostic and therapeutic purposes $(12,13)$. Even though this therapeutic modality has spread to the field of orofacial pain more than half a century ago (14), the number of clinical studies on TMJ is comparatively small. Kopp et al. examined the long-term clinical effects of intra-articular injections of betamethasone combined with lidocaine (15). It was reported that subjective symptoms and clinical signs in the injection group diminished compared to the occlusal treatment in a 2-year follow-up. A retrospective study by Samiee et al. reported the efficacy of intra-articular injections of triamcinolone combined with lidocaine in a 1-week follow-up (16). The average maximal mouth opening was significantly increased, but the pain intensity was not significantly different from pre-injection level. A clinical study of Gupta et al. compared the efficacy between hydrocortisone plus local anesthetic injection and PRP injection at follow-up of 1 and 12 weeks after injection (17). It showed that pain intensity gradually improved in both groups, but pain improvement in corticosteroid and local anesthetic injection group was inferior to those in the PRP group. Although intra-articular injections of corticosteroids and local anesthetics could be a possible treatment option for TMJ disorders, there is still room for discussion of its efficacy in the improvement of TMJ pain.

In terms of predictors for the improvement in TMJ pain, there are few studies available. Clinical studies on hyaluronic acid injections reported that potential predictive factors of treatment success are the affected side of arthritis, high baseline pain level (18), pain-related impairment, disability points (19), and degenerative changes on the pre-operative magnetic resonance imaging (MRI) (20). A clinical study on corticosteroid injection after arthrocentesis has failed to show potential predictors (21). To the best of the authors' knowledge, no information is available so far with regard to what factors can predict a better prognosis of corticosteroid injections in patients with TMJ disorders.

The objective of this study is to determine the efficacy of intra-articular injections of corticosteroid in combination with local anesthetic for pain relief in patients with TMJ pain and to identify predictive factors for pain improvement. The null hypothesis is that intra-articular injections of corticosteroid combined with local anesthetic do not affect TMJ pain improvement and there are no predictors of pain improvement. 
Efficacy of Intra-Articular Injection of Corticosteroids Combined with Local Anesthetics in the Management of Temporomandibular Joint Pain: A Retrospective Study

\section{MATERIALS AND MeTHODS}

\section{Study Population}

A retrospective study was conducted at the Orofacial Pain and Oral Medicine Center of the Herman Ostrow School of Dentistry of the University of Southern California. A review of electronic medical charts was performed by a single researcher on patients with the following characteristics: 1 ) persistent pain as the chief complaint, 2) tenderness of the TMJ with a palpation pressure of approximately $0.5 \mathrm{~kg}, 3$ ) diagnosed with TMJ disorders, such as osteoarthritis, disc disorders and concomitant muscle disorders, and 4) received intra-articular injections of corticosteroid combined with local anesthetic between February 2015 and August 2019. Written informed consent was obtained from all patients. Charts were excluded when patient did not return for a follow-up.

The diagnoses and treatments including the injections were provided by residents under the supervision of faculty following the protocol of the Orofacial Pain and Oral Medicine Center of Herman Ostrow School of Dentistry of USC based on the guidelines of the American Academy of Orofacial Pain (2) and the DC/ TMD criteria $(1,3)$. The differential diagnoses were labeled as primary, secondary, tertiary, and quaternary and were classified according to the 2020 release of the International Classification of Diseases, 10th Revision, Clinical Modification (ICD-10-CM). When a patient received injections on multiple visits, each injection case was included separately.

The study protocol followed the principles of the Declaration of Helsinki and was approved by the institutional review board at the University of Southern California (\# UP-07-00416).

\section{Treatment Procedure of Intra-Articular Injections}

Prior to the intra-articular corticosteroid injections, all patients were given counseling and home-based conservative physical care protocol, including avoidance of wide mouth opening and hard foods and mandibular rest position like [n] phonation with the tongue positioned in the anterior palate, along with jaw stretches. Medications were prescribed, if necessary.

Based on the diagnoses of TMDs, intra-articular injections were performed in the affected TMJs (16).
$1.5 \mathrm{ml}$ solution consisting of $0.5 \mathrm{ml}$ of triamcinolone acetonide (Kenalog ${ }^{\circledR}-40,40 \mathrm{mg} / \mathrm{ml}$, Bristol-Myers Squibb Company, Princeton, NJ, USA) and $1 \mathrm{ml}$ of $1 \%$ or $2 \%$ lidocaine hydrochloride (Xylocaine ${ }^{\circledR}, 10$ or 20 $\mathrm{mg} / \mathrm{ml}$, AstraZeneca Inc., Mississauga, ON, Canada) was prepared for intra-articular injection. The preauricular skin was cleaned with a $70 \%$ isopropyl alcohol pad. Patients were asked to open the mouth wide enough to locate the condyle of the TMJ. A 27gauge needle was inserted into the superior joint space, behind the condyle and beneath the zygoma, and passed in until three-fourths of the needle were in the joint space. The solution was injected into space after negative aspiration, with an ice pack applied to the TMJ area post-injection. Five minutes after the procedure, patients were assessed for their pain level and any complications including signs of facial palsy.

\section{Evaluation of Pain Intensity}

A verbal numerical rating scale (NRS) ranging from 0 to 10 was used to assess the pain intensity of the TMJs, on palpation of approximately $0.5 \mathrm{~kg}$ pressure (2). Patients were asked to rate the pain intensity using NRS for three times: 1) baseline before the injection on the day of treatment as NRS-BL, 2) immediately after the injection as NRS-IMM, and 3) at follow-up as NRSF/U. Based on these NRS scores, pain improvement between before and immediately after injection ( $\triangle$ IMM: NRS-BL minus NRS-IMM) and between pretreatment and post-treatment of the follow-up $(\Delta \mathrm{F} / \mathrm{U}$ : NRS-BL minus NRS-F/U) were calculated.

\section{Radiological Evaluation}

Digital panoramic radiograph images were obtained from the electronic medical charts in order to screen for degenerative changes in the condyle, assuming the actual clinical setting of the TMJ examination. When the panoramic images were included, they were analyzed independently by two blinded clinicians, who were calibrated previously on identifying radiological findings of the condyle, including normal, degenerative change and remodeling. The degenerative feature of the condyle is indicative of abnormalities in shape and form such as flattening of the condyles, loss of cortication, or erosions of the condyles. The remodeling feature is indicative of sclerotic changes with osteophyte formation. In cases of non-agreement with the findings, a radiology expert's advice (E. R.) was sought. 
Efficacy of Intra-Articular Injection of Corticosteroids Combined with Local Anesthetics in the Management of Temporomandibular Joint Pain: A Retrospective Study

\section{Statistical Analysis}

To investigate the efficacy of intra-articular injections, NRS-BL, NRS-IMM, and NRS-F/U were compared using a Friedman test, then Wilcoxon signed-rank tests were applied using the Bonferroni correction. A correlation coefficient between $\Delta \mathrm{F} / \mathrm{U}$ and the number of follow-up weeks was calculated to determine timedependent changes of pain NRS score.

Gender (female vs male), age (less than 51 vs not less than 51), diagnoses (presence of arthralgia, articular disc disorder, derangement, osteoarthritis, or masticatory myalgia vs absence), injection site (unilateral vs bilateral), medication (systemic NSAIDs vs others), and radiological findings (degenerative changes vs others) were considered as potential predictors for pain improvement at follow-up. To identify predictive factors, the effects of these variables on $\Delta \mathrm{F} / \mathrm{U}$ were analyzed using a Mann-Whitney's U test. Additionally, the correlation coefficient was calculated to investigate associations of both NRS-BL and $\triangle \mathrm{IMM}$ with $\Delta F / U$. Significant level $\alpha$ was set at 0.05 (JMP Pro version 15, SAS Institute Inc., Cary, NC, USA).

\section{RESULTS}

\section{Sample Profile}

A total of 45 injection cases were identified, and then 8 cases were excluded because of no-shows in the followup (no-show rate: 17.8\%). Thirty-seven injections of 31 patients including 25 females and 5 males were analyzed (Table 1). The age distribution showed that the group over 60 was more common. The initial chief complaint was pain for 21 cases, pain with joint sound for 9 cases, and pain with limited opening for 7 cases. Four patients received intra-articular injections two times, two of which were injected into the same side of TMJ. One patient received three injections; two for the right TMJ, one for the left TMJ. Identified diagnoses for the TMJ disorders in 37 patients were arthralgia (ICD-10-CM code: M26.62), articular disc disorder (M26.63), derangement (M26.69), and osteoarthritis (M19.90). The most common primary diagnosis was arthralgia (23 cases), followed by osteoarthritis (7 cases). The most common diagnosis concomitantly with diagnoses of the TMJ disorders was masticatory myalgia (M79.11). Cervical myalgia, myofascial pain, contracture, trismus, trigeminal neuropathy, sleep-related bruxism, obstructive sleep apnea, and tension-type headache were found in a few cases. 15 cases $(40.5 \%)$ were prescribed systemic NSAIDs including nabumetone and ibuprofen after injection. Degenerative changes of the injected condyle were found in 8 cases (27.0\%); however, no patient demonstrated remodeling of the condyle. In addition, clear panoramic radiographs were not available in 4 cases $(10.8 \%)$. The median follow-up period after injection was 5.0 weeks (range: 0.4-25.4).

Table 1. Demographic data

\begin{tabular}{|c|c|c|}
\hline \multicolumn{2}{|l|}{ Component } & $\mathrm{p}$-value \\
\hline \multicolumn{3}{|l|}{ Gender } \\
\hline \begin{tabular}{l|l} 
Female \\
\end{tabular} & 25 & \multirow{2}{*}{0.519} \\
\hline Male & 6 & \\
\hline \multicolumn{3}{|l|}{ Age } \\
\hline \begin{tabular}{l|l|} 
& $0-20$ \\
\end{tabular} & 4 & \multirow{6}{*}{0.915} \\
\hline $21-30$ & 4 & \\
\hline $31-40$ & 5 & \\
\hline $41-50$ & 6 & \\
\hline $51-60$ & 3 & \\
\hline$>60$ & 9 & \\
\hline \multicolumn{3}{|l|}{ Diagnosis } \\
\hline \begin{tabular}{l|l|} 
& Arthralgia of TMJ \\
\end{tabular} & 24 & 0.328 \\
\hline Articular disc disorder of TMJ & 10 & 0.629 \\
\hline Derangement of TMJ & 10 & 0.185 \\
\hline Osteoarthritis of TMJ & 11 & 0.004 \\
\hline $\begin{array}{l}\text { Myalgia of masticatory } \\
\text { muscle }\end{array}$ & 28 & 0.097 \\
\hline \multicolumn{3}{|l|}{ Injection site } \\
\hline \begin{tabular}{l|l} 
Unilateral \\
\end{tabular} & 36 & \multirow{2}{*}{0.925} \\
\hline Bilateral & 1 & \\
\hline \multicolumn{3}{|l|}{ Medication } \\
\hline $\begin{array}{l}\text { Systemic NSAID (ibuprofen, } \\
\text { nabumetone) }\end{array}$ & 15 & \multirow{5}{*}{0.513} \\
\hline Topical NSAID (ketoprofen) & 1 & \\
\hline Muscle relaxant (baclofen) & 1 & \\
\hline Analgesic (acetaminophen) & 1 & \\
\hline None & 19 & \\
\hline \multicolumn{3}{|c|}{ Radiological findings of condyle in the injection site } \\
\hline Normal & 25 & \multirow{3}{*}{0.515} \\
\hline Degenerative change & 8 & \\
\hline Clear image unavailable & 4 & \\
\hline
\end{tabular}

Archives of Anesthesiology V3 . I2 . 2020 
Efficacy of Intra-Articular Injection of Corticosteroids Combined with Local Anesthetics in the Management of Temporomandibular Joint Pain: A Retrospective Study

A total of 37 injection cases of 31 patients are shown. TMJ: temporomandibular joint. NSAID: nonsteroidal anti-inflammatory drug. Prior to statistical analysis, injection cases were divided into 2 groups as follows; age (less than 51 vs not less than 51), diagnosis (presence vs absence), medication (systemic NSAIDs vs others), and radiological findings (degenerative changes vs others). P-value represents a comparison of $\Delta \mathrm{F} / \mathrm{U}$ (improvement of pain score from baseline to follow-up) between the groups using a MannWhitney's U test. ${ }^{*} \mathrm{p}<0.05$.

\section{Efficacy f Intra-Articular Injections}

The median pain NRS score changed from 7 (NRS$\mathrm{BL}$, first quartile $=5$, third quartile $=8$, range: $2-10$ ) at baseline to 0 (NRS-IMM, first quartile $=0$, third quartile $=0$, range: $0-6$ ) immediately after injection, and then to $3(\mathrm{NRS}-\mathrm{F} / \mathrm{U}$, first quartile $=0$, third quartile $=6$, range: $0-8$ ) at follow-up. The improvement of pain NRS score was median 7 (first quartile $=4$, third quartile $=8$, range: $2-10$ ) for $\triangle \mathrm{IMM}$ and median 4 (first quartile $=1$, third quartile $=7$, range: $-1-10$ ) for $\Delta \mathrm{F} / \mathrm{U}$. If over $50 \%$ pain reduction (NRS-F/U $<50 \%$ of NRS-BL) is considered successful (22-24), 20 cases $(54.1 \%)$ achieved treatment success at follow-up. If over $30 \%$ pain reduction (NRS-F/U $<70 \%$ of NRS-BL) is considered successful, 23 cases $(62.2 \%)$ achieved success.

Friedman test demonstrated significant differences among NRS-BL, NRS-IMM, and NRS-F/U ( $p<0.001)$. Additionally, Wilcoxon signed-rank tests revealed that the pain NRS score significantly improved immediately after injections (Fig. 1, NRS-BL vs NRSIMM: $\mathrm{p}<0.001$ ) and at follow-up (NRS-BL vs NRS-F/U, $\mathrm{p}<0.001)$ compared with the baseline. At follow-up, pain increased significantly compared to the score recorded immediately after treatment (NRS-IMM vs NRS-F/U, $\mathrm{p}<0.001$ ). Considering the follow-up period, the improvement of pain NRS score at followup $(\Delta \mathrm{F} / \mathrm{U})$ was not significantly correlated with the number of follow-up weeks (Fig. 2, Spearman's rho: 0.022, $\mathrm{p}=0.896$, Kendall's tau: 0.016, $\mathrm{p}=0.895$ ).

With regard to the side effects of injections, two patients noticed transient facial palsy (5.4\%) and one patient showed a transient bite change (2.7\%), which returned to normal within a few minutes. All of the patients were discharged without any complications after injections.

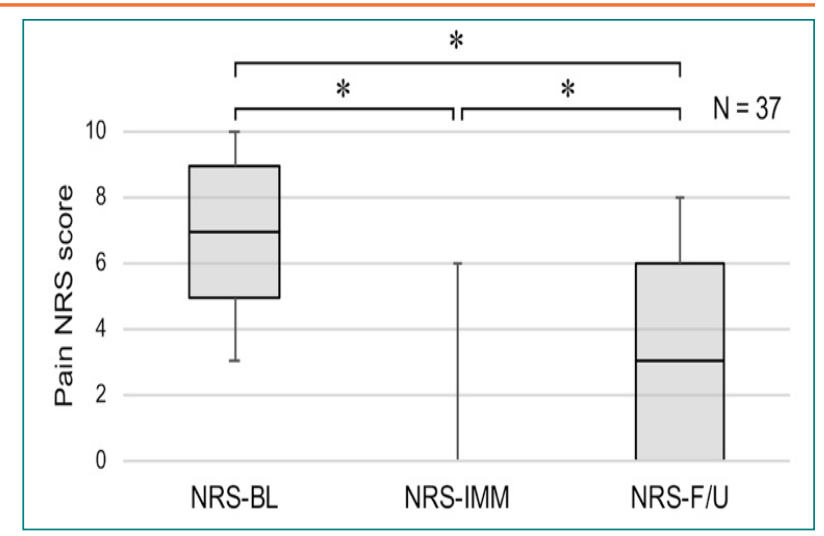

Figure 1. Changes of pain severity before and after intra-articular injections

The data are shown as the median and 1st and 3rd quartile. The whiskers represent minimum and maximum values. NRS: verbal numerical rating scale, NRS-BL: pain NRS score at baseline, NRS-IMM: pain NRS score immediately after injections, NRS-F/U: pain NRS score at follow-up. *p $<0.05$

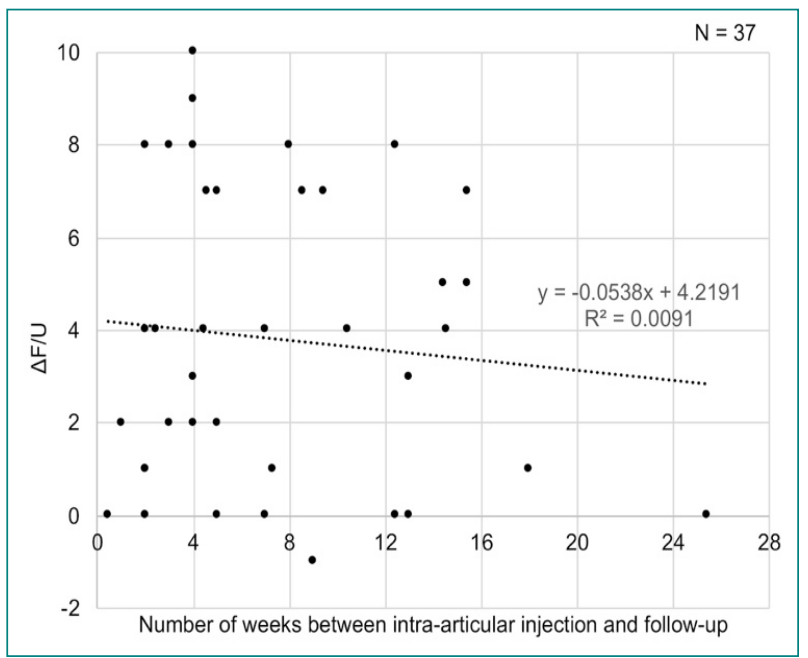

Figure 2. Time-dependent changes of $\Delta F / U$

$\Delta \mathrm{F} / \mathrm{U}$ : improvement of pain NRS (verbal numerical rating scale) score between the baseline and followup, higher $\Delta \mathrm{F} / \mathrm{U}$ represents greater improvement of TMJ pain. The line and formula represent a linear relationship between $\Delta \mathrm{F} / \mathrm{U}$ and follow-up weeks using a linear least squares regression.

\section{Correlations}

Mann-Whitney's U test revealed that patients without osteoarthritis were significantly associated with higher pain improvement of $\Delta \mathrm{F} / \mathrm{U}(\mathrm{p}=0.004)$ than those with osteoarthritis; however, no significant 
Efficacy of Intra-Articular Injection of Corticosteroids Combined with Local Anesthetics in the Management of Temporomandibular Joint Pain: A Retrospective Study

differences were found in the other variables ( $p>$ 0.05 , Table 1$)$. In addition, the baseline pain score of NRS-BL was significantly correlated with $\Delta \mathrm{F} / \mathrm{U}$ (Fig. 3, Spearman's rho: 0.328, $\mathrm{p}=0.048$, Kendall's tau: 0.275, $\mathrm{p}=0.033$ ). The immediate pain relief of $\triangle \mathrm{IMM}$ was also significantly correlated with $\Delta \mathrm{F} / \mathrm{U}$ (Fig. 4, Spearman's rho: $0.375, p=0.022$, Kendall's tau: 0.295, $p=0.020$ ).

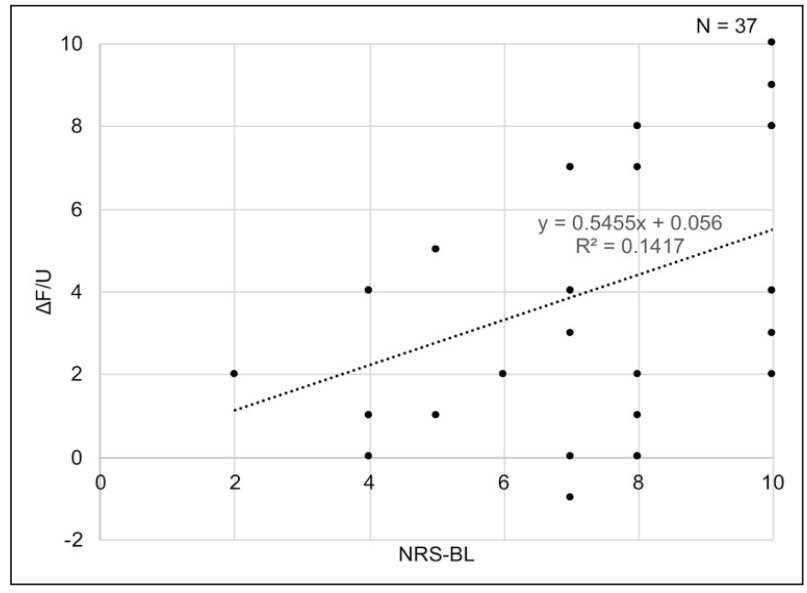

Figure 3. Correlation between $N R S-B L$ and $\Delta F / U$

NRS-BL: pain intensity assessed by a verbal numerical rating scale (NRS) at baseline, higher NRS-BL indicates greater pain of the TMJ. $\Delta \mathrm{F} / \mathrm{U}$ : improvement of pain NRS score between baseline and follow-up, higher $\Delta \mathrm{F} / \mathrm{U}$ indicates greater improvement. The line and formula represent a linear relationship between NRS$\mathrm{BL}$ and $\Delta \mathrm{F} / \mathrm{U}$ using a linear least squares regression.

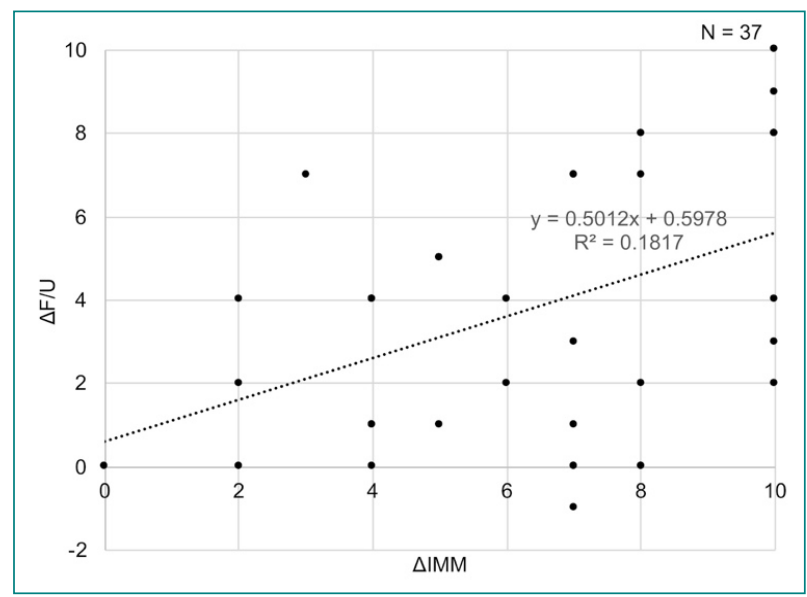

Figure 4. Correlation between $\triangle I M M$ and $\Delta F / U$

$\Delta \mathrm{IMM}$ : pain improvement assessed by a verbal numerical rating scale (NRS) from baseline to immediately after injections. $\Delta \mathrm{F} / \mathrm{U}$ : improvement of pain NRS score between the baseline and follow-up. Higher $\triangle \mathrm{IMM}$ and $\Delta \mathrm{F} / \mathrm{U}$ indicate greater improvement of TMJ pain from baseline. The line and formula represent a linear relationship between $\triangle \mathrm{IMM}$ and $\Delta \mathrm{F} / \mathrm{U}$ using a linear least squares regression.

\section{DISCUSSION}

This study showed that intra-articular injections of corticosteroids combined with local anesthetics drastically improved the pain intensity assessed by NRS immediately post-injection; however, transient facial palsy was observed in $5.4 \%$ of the patients and bite change occurred in $2.7 \%$, and all side effects were resolved within a few minutes. Although significant pain improvement was observed in response to injection, it was less significant at follow-up compared to immediately after injection, suggesting that local anesthetics temporarily played a predominant role in pain relief. Regarding predictive factors for pain improvement, patients without osteoarthritis tend to achieve better pain relief than those with osteoarthritis. Gender, age, injection site, medication, and radiological interpretations of the condyle failed to predict pain improvement. Furthermore, there was weak correlation between the improvement of pain NRS score at follow-up and both pre-injection pain NRS score and immediate pain relief after injection, suggesting that they can slightly predict improvement in pain NRS score. Therefore, the null hypothesis was rejected.

Corticosteroids possess anti-inflammatory properties, and lidocaine by itself can have some mild antiinflammatory effects during its short half-life of 1.5 hours $(12,13)$. In the current study, intra-articular injections of $20 \mathrm{mg}$ triamcinolone in combination with 10 or 20 mg lidocaine were significantly effective on pain relief in 37 patients with TMJ pain, the median pain NRS score changed from 7 at baseline to 3 at follow-up (approximately 60\% reduction, median follow-up period: 5.0 weeks). A retrospective study of Samiee et al. investigated the efficacy of intra-articular injections of $20 \mathrm{mg}$ triamcinolone combined with $20 \mathrm{mg}$ lidocaine on pain intensity in 17 patients with TMJ arthritis, capsulitis, or disc displacement without reduction with limited opening (16). The average pain score was reported to change from 8 to 4 in a 1-week follow-up (50\% reduction), and the change was not statistically significant. A clinical study of Gupta et al. evaluated pain intensity, mandibular range of motion, and joint sound in 14 patients with disc displacement 
Efficacy of Intra-Articular Injection of Corticosteroids Combined with Local Anesthetics in the Management of Temporomandibular Joint Pain: A Retrospective Study

with reduction when $0.5 \mathrm{ml}$ hydrocortisone plus 1.0 $\mathrm{ml}$ local anesthetic were injected (17). The average pain score seemed to change from about 3.5 at preinjection to 2.5 in 1-week follow-up (approximately $30 \%$ reduction), and then to 1.0 in 12 -week follow-up (approximately $70 \%$ reduction). Although the study population, initial pain level and duration of follow-up in the current study were different from those in the Samiee et al. and Gupta et al. studies, the results were similar in terms of pain reduction, and the sample size might have affected the statistical significance of the results. It should be noted that patients in the current study were also provided counseling and home-based conservative physical care protocol. Conservative therapy could enhance the therapeutic effectiveness of intra-articular injections in clinical context.

This study has revealed adverse effects of 5.4\% transient facial palsy and $2.7 \%$ transient bite change, lasting a few minutes after the injections. The development of facial paralysis and bite change could be induced by a mild pressure of the solution on the branch of the facial nerve (CN VII) or the mandibular nerve (V3) of the trigeminal nerve ( $\mathrm{CN} \mathrm{V}$ ) in proximity to the condyle. In these cases, the needle was probably not completely inserted into the joint space and the solution might have dissolved into the pre-auricular skin. A clinical study reported TMJ injection of triamcinolone mixed with lidocaine caused temporary facial palsy which persists for a few hours in 5.6\% of the sampled population (16), probably due to the anesthetic response of the facial nerve. Another study in 14 patients reported no adverse effects after TMJ injection of hydrocortisone mixed with local anesthetic (17). The adverse effects and its incidence in the current study were relatively common, considering the results of previous studies; clinical studies on TMJ intra-articular injection of sodium hyaluronate, PRP, or saline alone have reported discomfort at the injection site, localized pain and swelling on the day after injection, ear pressure, open bite, and generalized rashes as transient adverse effects, and have not reported any persistent side effects $(6,11,25-28)$. Regarding intra-articular corticosteroid injections into various joints, the possible side effects are postinjection pain and flare (1-10\%), facial flushing $(-15 \%)$, skin or fat atrophy (about $0.6 \%$ ), infection (0.002-0.033\%), temporary dizziness and syncope (about 1.3\%), joint destruction after repetitive injections, transient increases in blood glucose levels, and mild and transient suppression of the hypothalamic-pituitary-adrenal axis $(12,13,29-31)$. In most cases, adverse reactions occur later the same day, and post-injection discomfort could be delayed by 24 to 48 hours in approximately $10 \%$ of patients (29). Animal studies have suggested pieces of evidence on deleterious effects on articular cartilage and studies in humans have not shown similar results $(12,31)$, but many physicians recommend 12 weeks between injections of the same joint to avoid the risk of joint destruction (12). In the current study, three patients received two injections upon the same side of the TMJ, with a period of 24-150 weeks between first and second injections. Although the adverse effects in the present study were the same as previously reported, possible side effects should be considered when providing various treatment options to patients.

Regarding predictive factors for TMJ pain relief, the current study indicated that the improvement of pain NRS score at follow-up was significantly associated with the diagnosis without osteoarthritis. In addition, there were low correlations between the pain improvementand baseline pain NRS score and between the pain improvement and immediate pain relief after injection. Regardless of intra-articular injections, it was reported that the improvement of pain intensity in patients with TMDs is associated with the duration of the pain complaint related to TMDs, number of care providers, degree of functional impairment, and presence of depression, pain elsewhere, and parafunctional activities (32-35). A clinical study on arthrocentesis plus hyaluronic acid injections in patients with TMJ osteoarthritis demonstrated that the improvement of pain at chewing at the 3-month follow-up was significantly predicted by the affected side of arthritis and high baseline pain level (18). In addition, a clinical study on hyaluronic acid injections in patients with TMJ osteoarthritis reported that the change of pain at chewing was significantly predicted by pain-related impairment of the Graded Chronic Pain Scale and pain-related disability points (19). In spite of injectable agents, specific clinical conditions and baseline pain intensity possibly affect the improvement of TMJ pain after injections.

TMJ osteoarthritis is classified as one of the degenerative joint diseases according to the DC/TMD taxonomy $(1,3)$ and has a complex and multifactorial 
Efficacy of Intra-Articular Injection of Corticosteroids Combined with Local Anesthetics in the Management of Temporomandibular Joint Pain: A Retrospective Study

etiology. Metabolic and mechanical factors can contribute to the development of a sustained inflammatory process (36). The natural course of TMJ osteoarthritis can be divided into three slow progressive phases with periods of remission and cartilage regeneration; 1 ) the early phases for $2.5-4$ years associated with clicking sound and intermediate locking, 2) the intermediate phase for 0.5-1 year associated with joint pain, limited function, and grating sound, and 3) the late phase for 0.5 years without degenerative activity (37). Perhaps, most of the patients with osteoarthritis in the present study were in the intermediate phase and might show poor response to the therapy. On evaluating the radiographs of the degenerative joint diseases, flattening and cortical sclerosis are considered indeterminate findings and may represent normal variation, aging, or remodeling (2). Although no significant association was found between pain improvement and radiological findings of the condyle on panoramic radiographs in the present study, a clinical study of arthrocentesis and sodium hyaluronate injection in patients with disc displacement without reduction showed a predictor of degenerative changes on MRIs (20). Panoramic radiograph can provide information on dental and maxillofacial structures including the condyle, articular eminence, and articular fossa in a single image. However, morphologic features in panoramic imaging generally do not indicate TMDs (38) and are inferior to those in cone-beam computed tomography (CBCT) for assessment of bony changes and in MRI for assessment of articular disc location and morphology (39). Considering the significant association of osteoarthritis in the present study, the condyle morphology on CBCT imaging may be partially predictive for the outcome of intra-articular injections.

The most commonly used corticosteroids for intra-articular injections in rheumatologists were reported to be methylprednisolone acetate (45.0\%), triamcinolone acetonide (26.1\%), triamcinolone hexacetonide $(22.1 \%)$, and dexamethasone $(4.4 \%)$ (40). In the authors' university-based orofacial pain clinic, triamcinolone acetonide has been utilized because of the cost-effectiveness, anti-inflammatory potency, and duration of action (16). Most patients could not afford hyaluronic acid or PRP for injections, due to their cost and for not being covered by insurance in the United States. The triamcinolone acetonide has 3.2-6.4 days of serum half-life and an intermediateacting duration of approximately 14 days (31). The choice of injectable corticosteroids should be based on a variety of considerations, including the availability, cost, and pharmacokinetics of the agent.

A limitation of this study is that this is a retrospective study and not a randomized controlled trial. A comparison between corticosteroid mixed with local anesthetic and PRP has been reported (17), but further research will be needed to compare the efficacy between corticosteroid combined with local anesthetic and other agents. In addition, there were other concomitant conditions such as masticatory myalgia, which may have exacerbated the pain intensity of the TMJ due to the anatomic relation. Although the duration of follow-up was not correlated with the pain NRS score improvement in this study, a larger number of cases with a specified period of follow-up should be conducted to assess the outcome of injection in the future.

\section{CONCLUSION}

Within the frame of this study, intra-articular injections of corticosteroids in combination with local anesthetics have the potential for the pain management in patients with TMJ disorders, which may be predicted by the presence or absence of osteoarthritis. Corticosteroid plus local anesthetic injection does not pose serious adverse effects. The results highlight that pre-treatment conditions and diagnoses play a relevant role in the prognosis of TMJ pain after intra-articular injection.

\section{Acknowledgment}

The authors would like to thank Dr. Elham Radan, Director of Radiology Clinic at the Herman Ostrow School of Dentistry of the University of Southern California, for her radiological opinion/interpretation of the panoramic radiographs.

\section{Declaration of Interest Statement}

All authors declare that they have no known competing financial interests or personal relationships that could have appeared to influence the work reported in this paper.

\section{Author Contribution Statement}

All authors have contributed to the acquisition and interpretation of the data and critically revised the 
Efficacy of Intra-Articular Injection of Corticosteroids Combined with Local Anesthetics in the Management of Temporomandibular Joint Pain: A Retrospective Study

manuscript. All authors approved the final version of the manuscript and agreed to be accountable for all aspects of the work in ensuring that questions related to the accuracy or integrity of any part of the work are appropriately investigated and resolved.

Funding: None.

\section{REFERENCES}

[1] Schiffman E, Ohrbach R, Truelove E, et al. Diagnostic Criteria for Temporomandibular Disorders (DC/TMD) for Clinical and Research Applications: recommendations of the International RDC/TMD Consortium Network* and Orofacial Pain Special Interest Group†. J Oral Facial Pain Headache. 2014;28(1):6-27. Available from: doi:10.11607/jop.1151

[2] de Leeuw R, Klasser GD. Orofacial Pain: Guidelines for Assessment, Diagnosis, and Management. 6th ed. Batavia, IL, USA: Quintessence Publishing Co.; 2018.

[3] Peck CC, Goulet J-P, Lobbezoo F, et al. Expanding the taxonomy of the diagnostic criteria for temporomandibular disorders. J Oral Rehabil. 2014;41(1):2-23. Available from: doi:10.1111/ joor.12132

[4] Murphy MK, MacBarb RF, Wong ME, Athanasiou KA. Temporomandibular disorders: a review of etiology, clinical management, and tissue engineering strategies. Int $J$ Oral Maxillofac Implants. 2013;28(6):e393-414. Available from: doi:10.11607/jomi.te20

[5] Liu Y, Wu J-S, Tang Y-L, Tang Y-J, Fei W, Liang $\mathrm{X}-\mathrm{H}$. Multiple treatment meta-analysis of intraarticular injection for temporomandibular osteoarthritis. J Oral Maxillofac Surg. 2020;78(3):373.e1-373.e18. Available from: doi:10.1016/j.joms.2019.10.016

[6] Moldez MA, Camones VR, Ramos GE, Padilla M, Enciso R. Effectiveness ofintra-articularinjections of sodium hyaluronate or corticosteroids for intracapsular temporomandibular disorders: a systematic review and meta-analysis. J Oral Facial Pain Headache. 2018;32(1):53-66. Available from: doi:10.11607/ofph.1783

[7] Liu Y, Wu J, Fei W, et al. Is there a difference in intra-articular injections of corticosteroids, hyaluronate, or placebo for temporomandibular osteoarthritis? J Oral Maxillofac Surg. 2018;76(3):504-514. Available from: doi:10.1016/j.joms.2017.10.028

[8] Manfredini D, Piccotti F, Guarda-Nardini L. Hyaluronic acid in the treatment of TMJ disorders: a systematic review of the literature. Cranio. 2010;28(3):166-176. Available from: doi:10.1179/crn.2010.023

[9] Al-Hamed FS, Hijazi A, Gao Q, Badran Z, Tamimi F. Platelet concentrate treatments for temporomandibular disorders: a systematic review and meta-analysis. To be published in JDR Clin Transl Res. [Preprint] 2020. Available from: doi:10.1177/2380084420927326

[10] Bousnaki M, Bakopoulou A, Koidis P. Plateletrich plasma for the therapeutic management of temporomandibular joint disorders: a systematic review. Int $J$ Oral Maxillofac Surg. 2018;47(2):188-198. Available from: doi:10.1016/j.ijom.2017.09.014

[11] Li F, Wu C, Sun H, Zhou Q. Effect of platelet-rich plasma injections on pain reduction in patients with temporomandibular joint osteoarthrosis: a meta-analysis of randomized controlled trials. $J$ Oral Facial Pain Headache. 2020;34(2):149-156. Available from: doi:10.11607/ofph.2470

[12] Lavelle W, Lavelle ED, Lavelle L. Intra-articular injections. Med Clin North Am. 2007;91(2):241250. doi:10.1016/j.mcna.2006.12.002

[13] SchumacherHR.Aspirationandinjectiontherapies for joints. Arthritis Rheum. 2003;49(3):413-420. Available from: doi:10.1002/art.11056

[14] Wenneberg B, Kopp S. Short term effect of intra-articular injections of a corticosteroid on temporomandibular joint pain and dysfunction. Swed Dent J. 1978;2(6):189-196.

[15] Kopp S, Wenneberg B. Effects of occlusal treatment and intraarticular injections on temporomandibular joint pain and dysfunction. Acta Odontol Scand. 1981;39(2):87-96. Available from: doi:10.3109/00016358109162265

[16] Samiee A, Sabzerou D, Edalatpajouh F, Clark GT, 
Efficacy of Intra-Articular Injection of Corticosteroids Combined with Local Anesthetics in the Management of Temporomandibular Joint Pain: A Retrospective Study

Ram S. Temporomandibular joint injection with corticosteroid and local anesthetic for limited mouth opening. J Oral Sci. 2011;53(3):321-325. Available from: doi:10.2334/josnusd.53.321

[17] Gupta S, Sharma AK, Purohit J, Goyal R, Malviya Y, Jain S. Comparison between intraarticular platelet-rich plasma injection versus hydrocortisone with local anesthetic injections in temporomandibular disorders: a double-blind study. Natl J Maxillofac Surg. 2019;9(2):205-208. Available from: doi:10.4103/njms.NJMS_69_16

[18] Guarda-Nardini L, Ferronato G, Favero L, Manfredini D. Predictive factors of hyaluronic acid injections short-term effectiveness for TMJ degenerative joint disease. $J$ Oral Rehabil. 2011;38(5):315-320. Available from: doi:10.1111/j.1365-2842.2010.02164.x

[19] Manfredini D, Favero L, Del Giudice A, Masiero S, Stellini E, Guarda-Nardini L. Axis II psychosocial findings predict effectiveness of TMJ hyaluronic acid injections. Int J Oral Maxillofac Surg. 2013;42(3):364-368. Available from: doi:10.1016/j.ijom.2012.10.033

[20] Aktas I, Yalcin S, Sencer S. Prognostic indicators of the outcome of arthrocentesis with and without sodium hyaluronate injection for the treatment of disc displacement without reduction: a magnetic resonance imaging study. Int J Oral Maxillofac Surg. 2010;39(11):1080-1085. Available from: doi:10.1016/j.ijom.2010.07.001

[21] Tabrizi R, Karagah T, Arabion H, Soleimanpour MR, Soleimanpour M. Outcomes of arthrocentesis for the treatment of internal derangement pain: with or without corticosteroids? J Craniofac Surg. 2014;25(6):e571-e575. Available from: doi:10.1097/SCS.0000000000001168

[22] Farrar JT, Dworkin RH, Max MB. Use of the cumulative proportion of responders analysis graph to present pain data over a range of cut-off points: making clinical trial data more understandable. J Pain Symptom Manage. 2006;31(4):369-377. Available from: doi:10.1016/j.jpainsymman.2005.08.018

[23] Dworkin RH, Turk DC, Wyrwich KW, et al. Interpreting the clinical importance of treatment outcomes in chronic pain clinical trials: IMMPACT recommendations. J Pain. 2008;9(2):105-121. Available from: doi:10.1016/j.jpain.2007.09.005

[24] Haythornthwaite JA. IMMPACT recommendations for clinical trials: opportunities for the RDC/ TMD. J Oral Rehabil. 2010;37(10):799-806. doi:10.1111/j.1365-2842.2010.02152.x

[25] Bertolami CN, Gay T, Clark GT, et al. Use of sodium hyaluronate in treating temporomandibular joint disorders: a randomized, double-blind, placebo-controlled clinical trial. J Oral Maxillofac Surg. 1993;51(3):232-242. Available from: doi:10.1016/s0278-2391(10)80163-6

[26] Bjørnland T,Gjærum AA, MøystadA. Osteoarthritis of the temporomandibular joint: an evaluation of the effects and complications of corticosteroid injection compared with injection with sodium hyaluronate. J Oral Rehabil. 2007;34(8):583589. Available from: doi:10.1111/j.13652842.2007.01759.x

[27] Hancı M, Karamese M, Tosun Z, Aktan TM, Duman $S$, Savaci N. Intra-articular platelet-rich plasma injection for the treatment of temporomandibular disorders and a comparison with arthrocentesis. J Craniomaxillofac Surg. 2015;43(1):162-166. Available from: doi:10.1016/j.jcms.2014.11.002

[28] Hegab AF, Ali HE, Elmasry M, Khallaf MG. Plateletrich plasma injection as an effective treatment for temporomandibular joint osteoarthritis. $J$ Oral Maxillofac Surg. 2015;73(9):1706-1713. Available from: doi:10.1016/j.joms.2015.03.045

[29] Kumar N, Newman RJ. Complications of intraand peri-articular steroid injections. $\mathrm{Br} J \mathrm{Gen}$ Pract. 1999;49(443):465-466.

[30] Charalambous CP, Tryfonidis M, Sadiq S, Hirst P, Paul A. Septic arthritis following intra-articular steroid injection of the knee--a survey of current practice regarding antiseptic technique used during intra-articular steroid injection of the knee. Clin Rheumatol. 2003;22(6):386-390. Available from: doi:10.1007/s10067-0030757-7

[31] Cole BJ, Schumacher HR. Injectable corticosteroids in modern practice. J Am Acad Orthop Surg. 2005;13(1):37-46. Available from: doi:10.5435/00124635-200501000-00006 
Efficacy of Intra-Articular Injection of Corticosteroids Combined with Local Anesthetics in the Management of Temporomandibular Joint Pain: A Retrospective Study

[32] Rollman A, Visscher CM, Gorter RC, Naeije M. Improvement in patients with a TMD-pain report. A 6-month follow-up study. J Oral Rehabil. 2013;40(1):5-14. Available from: doi:10.1111/ joor.12009

[33] Ohrbach R, Slade GD, Bair E, et al. Premorbid and concurrent predictors of TMD onset and persistence. Eur J Pain. 2020;24(1):145-158. Available from: doi:10.1002/ejp.1472

[34] Osiewicz M, Lobbezoo F, Ciapała B, PytkoPolończyk J, Manfredini D. Pain predictors in a population of temporomandibular disorders patients. J Clin Med. 2020;9(2):452. Available from: doi: $10.3390 / \mathrm{jcm} 9020452$

[35] Su N, Lobbezoo F, van Selms MKA, van der Heijden GJMG. Development and internal validation of prediction models for persistence of self-reported orofacial pain in the follow-up of patients with myofascial pain. To be published in Clin Oral Investig. [Preprint] 2020. Available from: doi:10.1007/s00784-020-03570-4

[36] Tanaka E, Detamore MS, Mercuri LG. Degenerative disorders of the temporomandibular joint: etiology, diagnosis, and treatment. J Dent Res. 2008;87(4):296-307. Available from: doi:10.1177/154405910808700406
[37] Kalladka M, Quek S, Heir G, Eliav E, Mupparapu M, Viswanath A. Temporomandibular joint osteoarthritis: diagnosis and long-term conservative management: a topic review. J Indian Prosthodont Soc. 2014;14(1):6-15. Available from: doi:10.1007/s13191-013-0321-3

[38] Crow HC, Parks E, Campbell JH, Stucki DS, Daggy J. The utility of panoramic radiography in temporomandibular joint assessment. Dentomaxillofac Radiol. 2005;34(2):91-95. Available from: doi:10.1259/dmfr/24863557

[39] Hussain AM, Packota G, Major PW, FloresMir C. Role of different imaging modalities in assessment of temporomandibular joint erosions and osteophytes: a systematic review. Dentomaxillofac Radiol. 2008;37(2):63-71. Available from: doi:10.1259/dmfr/16932758

[40] Lazaro D, Alon L, Ramessar N, Cabas-Vargas J, Shwin K, Stefanov DG. Intra-articular, bursa, and tendon sheath injections: a survey of practice patterns among members of the American College of Rheumatology. J Clin Rheumatol. 2014;20(2):91-93. Available from: doi:10.1097/ RHU.0000000000000080

Citation: Chitra Priya Emperumal, Mariela Padilla, et al. Efficacy of Intra-Articular Injection of Corticosteroids Combined with Local Anesthetics in the Management of Temporomandibular Joint Pain: A Retrospective Study. Archives of Anesthesiology. 2020; 3(2): 31-41.

Copyright: (C) 2020: Chitra Priya Emperumal, Mariela Padilla, et al. This is an open access article distributed under the Creative Commons Attribution License, which permits unrestricted use, distribution, and reproduction in any medium, provided the original work is properly cited. 